\title{
Understanding Fundamental Processes in Carbon Materials with Well-Defined Colloidal Graphene Quantum Dots
}

\author{
Qiqi Li, Benjamin W. Noffke, Yijun Liu, Liang-shi Li* \\ Department of Chemistry, Indiana University, Bloomington Indiana USA 47405
}

\begin{abstract}
Carbon materials have played very important roles in our society. Due to their complexity and inhomogeneity, however, our understandings of these materials have seriously lagged behind. As a result, development of new carbon materials has been largely based on trial and error. Here we review our recent work on using well-defined colloidal graphene quantum dots as model systems to study fundamental processes in carbon materials. Future directions and limitations of this approach are also discussed.
\end{abstract}

* To whom correspondence should be addressed. Email: $\underline{\text { li23@indiana.edu }}$ 


\section{Introduction}

It is impossible to overestimate the importance of carbon materials in our society. Indeed they are ubiquitous, ranging from lead in pencils, carbon black in tires, to adsorbents in gas masks and electrodes in batteries. In addition, carbon constitutes a large portion of energy supply we have today (i.e., coal), yet with the unfortunate side effect of releasing a large amount of $\mathrm{CO}_{2}$ to the atmosphere. Driven by concerns about the adverse impact due to human activities and demands for technologies to enable global sustainability, research efforts have intensified in recent years to broaden the use of carbon materials. Important directions include developing new carbon materials for renewable energy applications and to carry out functions that traditionally require rare and precious metals. ${ }^{[1-3]}$

In sharp contrast to the wide applications of the graphitic carbon materials is our limited and mostly empirical understanding of fundamental processes in them, primarily because of the heterogeneity and complexity of these materials. As a result, development of new carbon materials has been largely based on trial and error. A molecular understanding will undoubtedly facilitate the advancement, which however demands a model system to study these complex materials.

Can graphene, a single atomic layer of graphite, provide a natural model system for studying the graphitic carbon materials? After all the graphitic carbon materials, such as graphite, carbon black, activated carbon, carbon fibers, and nanotubes, overwhelmingly are made of conjugated framework of $\mathrm{sp}^{2}$-hybridized carbon atoms. And single-domains of graphene up to millimeters in size can be readily isolated through mechanical exfoliation and can be studied with various experimental techniques. However, graphene has a few intrinsic disadvantages. For example, it has been demonstrated that either the support underneath $^{4}$ or mechanical strain $^{[5,6]}$ can significantly alter electronic properties of graphene and its chemical reactivity. In addition, graphene consists of only surface and in essence a single molecule. This means that to study chemical processes involving graphene requires sophisticated techniques with high sensitivity. Further, many processes occurring in the graphitic carbon materials involve heteroatoms. The functionalization (or doping) of graphene is mostly achieved with high temperature, plasma, or strong oxidants. ${ }^{[7-9]}$ The harsh doping conditions and poorly controlled chemistry result in defects and are not capable of controlling the location, density, or bonding configurations of the heteroatoms, making accurate characterization of the materials difficult. The same difficulties 
also exist in doping of carbon nanotubes or graphite, and thus alternatives are needed as model systems for the graphitic carbon materials.

In this perspective, we discuss recent work in our research group that uses well-defined colloidal graphene quantum dots $(\mathrm{GQDs})^{[10,11]}$ as model systems to understand fundamental processes in graphitic carbon materials. We will first briefly introduce the GQDs, followed by their applications in studying carbon materials for catalysis and mechanisms of chemical reactions in carbon materials.

\section{Why Graphene Quantum Dots}

The graphene quantum dots (GQDs) discussed in this perspective have well-defined structures and are soluble (dispersible) in solvents (examples in Figure 1). ${ }^{[12]}$ They are synthesized from small aromatic compounds through stepwise solution chemistry (e.g., Figure 2), and have excellent size uniformity. To make them soluble in common solvents such as chloroform, toluene, and so forth, we recently developed a new solubilization strategy for large polycyclic aromatic hydrocarbons. ${ }^{[13,14]}$ This was achieved by covalently attaching multiple $2^{\prime}, 4^{\prime}, 6^{\prime}$-trialkyl-substituted phenyl moieties (at the 1'-position) to the edges of graphene (Figure
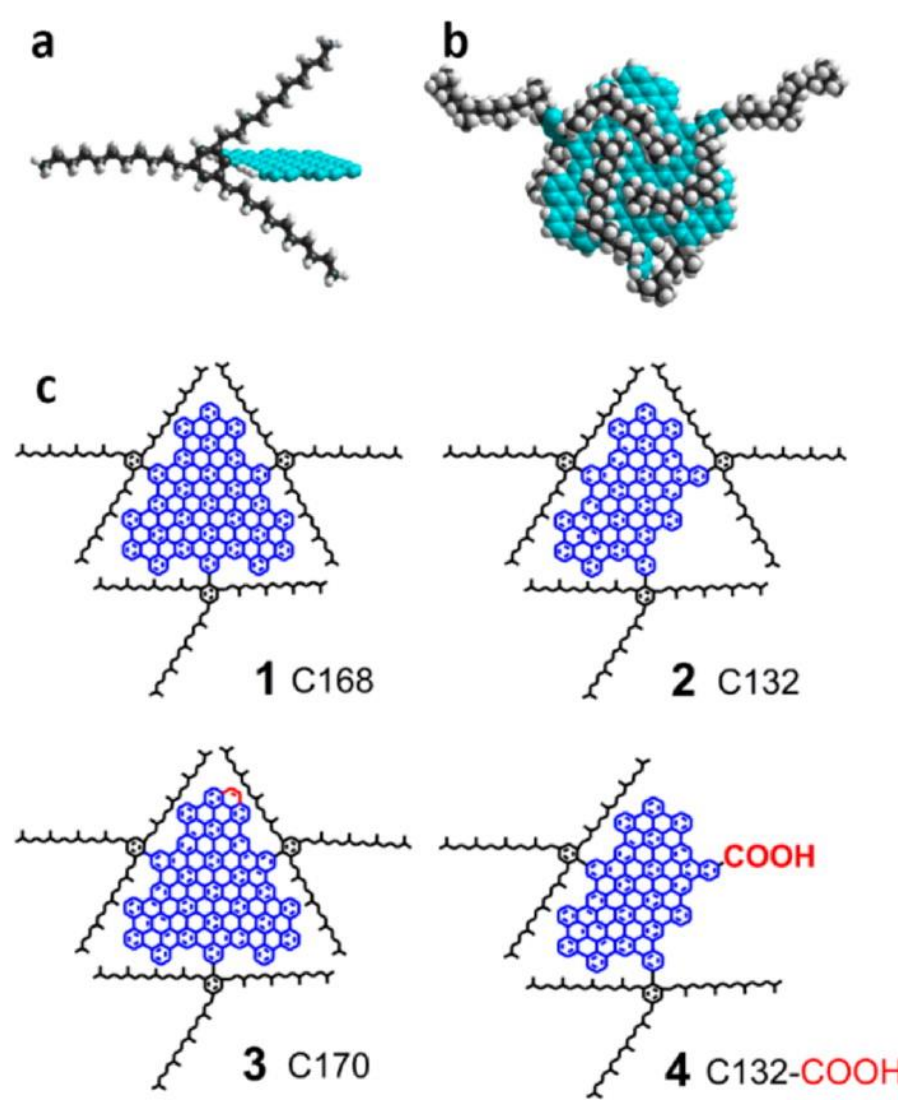

Figure 1. Colloidal graphene quantum dots (GQDs) with well-defined structures. (a) A 2', 4',6'-trialkylphenyl moiety (black) is covalently attached to the edge of the graphene (blue) so that the crowdedness on the edge forces the peripheral phenyl groups to twist from the plane of the graphene. (b) An energy-minimized geometry of GQD 1 (in c), showing the three-dimensional cage made of the flexible alkyl chains (black) around the graphene core (blue). (c) Structures of examples of colloidal GQDs, with the graphene core containing 168, 132, 170, and 132 conjugated carbon atoms. The structures of the GQDs are controlled with stepwise solution chemistry (Figure 2) so that they have excellent size uniformity. (Adapted with permission from ref. 10. Copyright 2013 American Chemical Society.) 
1a). The crowding on the edges forces the peripheral phenyl groups to twist from the plane of the graphene, resulting in the alkyl chains forming a three-dimensional "cage" around it (Figure 1b). This leads to increased distance between the conjugated systems in all three dimensions and thus greatly reduces the intermolecular $\pi-\pi$ attraction. The new solubilization approach enabled us to synthesize colloidal graphene quantum dots (GQDs) with well-defined molecular structures, e.g., 1-4 in Figure 1c that contain 168, 132, 170, and 132 conjugated carbon atoms in the graphene core (marked blue), respectively.

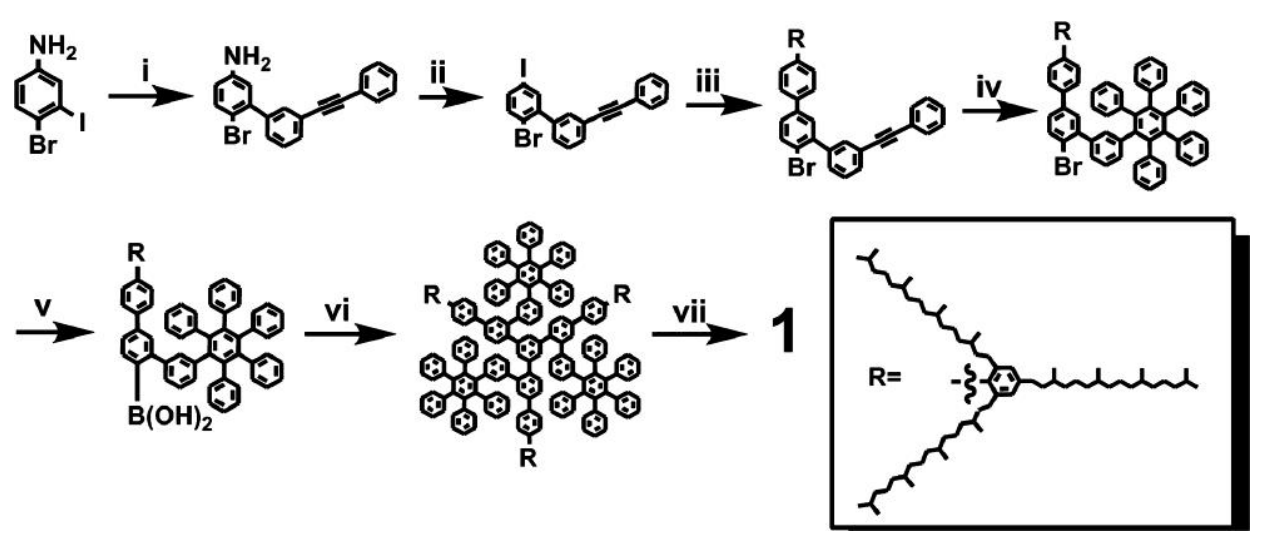

The well-defined GQDs have some unique

characteristics that make them excellent model systems for studying

Figure 2. Synthetic route for colloidal GQD 1. The GQD is synthesized with stepwise organic reactions and has excellent size uniformity. Conditions: (i) 3(phenylethynyl)phenylboronic acid, $\mathrm{Pd}\left(\mathrm{PPh}_{3}\right)_{4}, \mathrm{~K}_{2} \mathrm{CO}_{3}$, toluene, $\mathrm{EtOH}, \mathrm{H}_{2} \mathrm{O}$, $80{ }^{\circ} \mathrm{C}$ (94\%); (ii) $\mathrm{I} 2$, $t$-butyl nitrite, benzene, $5{ }^{\circ} \mathrm{C}$ (54\%); (iii) 4-(2',4',6'trialkylphenyl)phenylborate, $\mathrm{Pd}\left(\mathrm{PPh}_{3}\right)_{4}, \mathrm{~K}_{2} \mathrm{CO}_{3}$, toluene, EtOH, $\mathrm{H}_{2} \mathrm{O}, 80{ }^{\circ} \mathrm{C}$ (92\%); (iv) tetraphenylcyclopentadienone, diphenyl ether, reflux (61\%); (v) (a) $n$-BuLi, THF, $-78{ }^{\circ} \mathrm{C}$, (b) $\mathrm{B}(i-\mathrm{PrO})_{3}$, (c) $\mathrm{HCl}, \mathrm{H}_{2} \mathrm{O}$ (60\%); (vi) 1,3,5triiodobenzene, $\mathrm{Pd}\left(\mathrm{PPh}_{3}\right)_{4}, \mathrm{~K}_{2} \mathrm{CO}_{3}, \mathrm{H}_{2} \mathrm{O}$, toluene, $80{ }^{\circ} \mathrm{C}$ (52\%); (vii) $\mathrm{FeCl}_{3}$, $\mathrm{CH}_{2} \mathrm{Cl}_{2}, \mathrm{CH}_{3} \mathrm{NO}_{2}$ (100\%). (Adapted with permission from ref 10. Copyright 2013 American Chemical Society.)

fundamental

processes in complex carbon materials. Clearly, the structural uniformity of the GQDs is matched by few other carbon nanostructures we know so far (i.e., fullerenes and nanotubes); yet the GQDs enjoy much greater structural tunability because they can be made with various sizes, shape, and symmetry. As we will see below, the solution chemistry synthesis further allows us to incorporate heteroatoms necessary for some applications into the conjugated framework with atomic precision, which has been very challenging for other carbon nanostructures. The high solubility of the GQDs in common solvents is also very important for experimental studies. It enables us to apply readily available, versatile ensemble characterization techniques, which together with the structural uniformity can greatly simplify interpretation of experimental results. 
What we believe will be particularly important and fruitful in carbon research is that the GQDs enable direct comparison between experiments and computational studies. Using nanoflakes as computational models for carbon materials have gained popularity in recently years due to the advances in theoretical methods and availability of more powerful computers. However, to model carbon materials various assumptions have to be made regarding the size of the nanoflakes, positions or bonding of dopants, etc., whereas the well-defined GQDs make such assumption unnecessary. As will be seen in the work discussed below, the direct comparison between experimental and computational studies has been crucial in our studies to reveal active intermediates and propose possible reaction pathways.

\section{Study Carbon Materials for Catalysis}

\subsection{Carbon-Metal Interactions}

The unique features of the well-defined GQDs together with their large sizes make them excellent model systems to study the interaction between the conjugated carbon framework and metal nanoparticles. Graphitic carbon materials, including cokes, active carbon, carbon black, carbon fibers, and nanotubes, are often used to as the supports for metal nanoparticles in catalysis. ${ }^{[15,16]}$ However, because of the inhomogeneity and complexity of these carbon materials, our knowledge on metal-carbon interactions comes mainly from theoretical calculations, and direct comparison between experiments and the calculations has been very challenging. ${ }^{[17,18]}$ For example, it is generally believed that oxygen-containing functionalities, such as carboxylic, carbonyl, and phenolic groups, are necessary for anchoring of metal nanoparticles on the carbon supports. ${ }^{[19-21]}$ Theoretical calculations, however, have shown considerable affinity between some metals and pristine graphene or carbon nanotubes. ${ }^{[22,23]}$

We used colloidal GQD 2 (in Figure 1) as a model system for carbon materials to probe the interactions between carbon and palladium particles. ${ }^{[24]}$ Palladium is interesting because $\mathrm{Pd}$ particles on carbon support are widely used in heterogeneous catalysis and $\mathrm{Pd}$ was found to form reproducible ohmic contacts with carbon nanotubes and graphene. ${ }^{[25,26]}$ In addition, interaction between Pd particles and various carbon supports has been extensively studied. DFT calculations of Pd atoms or clusters adsorbed on graphene have shown significant mixing between the Pd $4 \mathrm{~d}$ orbitals and the $\pi$-orbitals of graphene through electron donation and back-donation. ${ }^{[23]}$ This indicates a covalent interaction, which however was not experimentally confirmed before. 
We synthesized palladium nanoparticles in the presence of the GQDs, and found that the Pdgraphene affinity was sufficient to stabilize the nanoparticles and prevent their aggregation. ${ }^{[2]}$ The covalent nature of the interaction was confirmed with infrared (IR) spectroscopy. Because of the well-defined structure of the GQDs, ensemble IR measurements could be easily carried out and interpreted. Figure 3 shows the comparison between the IR spectra of the GQDs in regions characteristic of aromatic $\mathrm{C}=\mathrm{C}$ stretching $\left(\sim 1600 \mathrm{~cm}^{-1}\right)$ and $\mathrm{C}-\mathrm{H}$ out-of-plane bending modes (between 700 and $1000 \mathrm{~cm}^{-1}$ ) before and after the Pd particles were made (black and red curves,

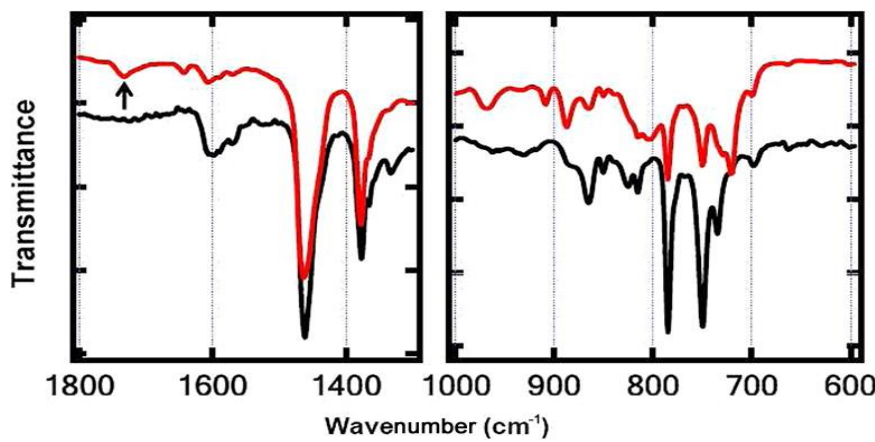

Figure 3. IR absorption spectra of the Pd/graphene mixtures (red curve) and $\mathbf{2}$ (black curve) in selected energy ranges. The spectra were normalized in intensity relative to the aliphatic $\mathrm{C}-\mathrm{H}$ deformation at 1377 and $1460 \mathrm{~cm}-1$. The peak at $1730 \mathrm{~cm}-1$, marked by an arrow, is due to the overtone (or combination) of aromatic $\mathrm{C}-\mathrm{H}$ out-of-plane bending modes. The changes in the aromatic $\mathrm{C}=\mathrm{C}$ stretching and $\mathrm{C}-\mathrm{H}$ out-of-plane bending regions indicate the covalent nature of the interaction between graphene and Pd nanoparticles. (Adapted with permission from ref 24. Copyright 2012 American Chemical Society.) respectively). With the intensities normalized relative to the aliphatic $\mathrm{C}-\mathrm{H}$ deformation (1377 and $1460 \mathrm{~cm}^{-1}$ ), the $\mathrm{C}=\mathrm{C}$ stretching absorption reduces significantly in intensity, suggesting the chemical interaction between the $\pi$ electrons in the graphene and the $\mathrm{Pd}$ particles. However, no obvious shift in the $\mathrm{C}=\mathrm{C}$ stretching frequency is observed, indicating the little disturbance in the $\mathrm{C}=\mathrm{C}$ bonds, consistent with the small changes in the UV-vis spectrum of $2 .^{24}$ The chemical interaction between the GQDs and the Pd nanoparticles is further confirmed by the spectral changes between 700 and 1000 $\mathrm{cm}^{-1}$ where many new peaks appear and the original peaks reduce in intensity. Thus we concluded that the Pd-graphene interaction

must be covalent, for the first time providing direct experimental evidence for previous theoretical predictions.

\subsection{Carbon as Catalysts}

Graphitic carbon materials themselves are very attractive catalysts for some very important chemical reactions. $\left.{ }^{[27}\right]$ To give some examples, activated cokes have been commercially applied to simultaneously remove $\mathrm{SO}_{2}$ and $\mathrm{NO}_{x}$ from flue gases in coal power plants. ${ }^{[28]}$ Surface- 
modified carbon nanotubes catalyze oxidative dehydrogenation of hydrocarbons with selectivity superior to state-of-the-art metal catalysts. ${ }^{[29,30]}$ More recently, heteroatom-doped carbon has drawn enormous interest because it can catalyze reactions such as the oxygen reduction (ORR) in fuel cells that typically require platinum. ${ }^{[1,2]}$ However, mechanistic studies on carbon catalysis have been severely hindered by their inhomogeneity and complexity. A molecular-level understanding of the carbon catalysis will undoubtedly facilitate discoveries of new carbon catalysts with improved reactivity and selectivity.

Understanding the roles of heteroatoms holds the key to understanding the catalytic mechanisms of carbon materials. Introducing heteroatoms into carbon materials, however, is generally an uncontrolled process, leading to an extra level of complexity due to various locations and binding configurations the dopants can adopt. ${ }^{[1,2,8]}$ For example, oxygen dopants are almost always present in graphic carbon materials, with oxygen atoms in functional groups varying from hydroxyl to carbonyl and acids. N-doping of carbon materials is achieved typically through pyrolysis or chemical vapor deposition, resulting in materials with very complicated morphologies and the nitrogen dopants in various bonding configurations. ${ }^{[1,2]}$ As a result, it hasn't been possible to fully characterize these materials, nor is it clear what parameters dictate their catalytic properties. For the ORR electrocatalyzed by the N-doped carbon, for example, the bonding configuration of the $\mathrm{N}$ dopants is traditionally considered to be the predominant parameter, and it has been characterized solely by the binding energy of the $\mathrm{N} 1$ s orbital obtained with X-ray photoelectron spectroscopy (XPS). ${ }^{[1,2]}$ Consequently, the nitrogen dopants are commonly categorized as, for example, "pyrrolic", i.e., those in a five-membered aromatic ring, "pyridinic", broadly defined as those in a six-membered aromatic ring and bonding to two carbon atoms, "quaternary", in a six-membered aromatic ring and bonding to three carbon atoms, and others. Now it is generally agreed that either the pyridinic or the quaternary nitrogen atoms are responsible for the ORR. As a result, increasing the nitrogen content with either configuration has been the major strategy to obtain better ORR catalysts, leading to conflicting reports and persisting debates regarding which of the two configurations is more effective. Further, the XPS approach itself has serious limitations - it can't distinguish the nitrogen dopants by their molecular environments. For example, the "pyridinic" nitrogen atoms characterized by the $\mathrm{N}$ 1s binding energy can appear in various nitrogen-containing functionalities such as pyridine, pyrazine, phenazine, triazine, etc., which are known to have different chemical 
properties yet have been treated indiscriminately by the carbon catalysis community. ${ }^{[31]}$ The roles of the oxygen dopants cannot be evaluated either. Unless it is directly bonded to a nitrogen atom, XPS gives no indication of an oxygen atom nearby.

We recently embarked on synthesizing $\mathrm{N}$-doped colloidal GQDs to study the structural dependence of the N-dopants in ORR catalysis. ${ }^{[32]}$ By combining the solubilization method we developed with solution chemistry, we synthesized GQDs of various sizes doped with nitrogen atoms (5-7, Figure 4). The GQDs all have two nitrogen atoms with a controlled bonding configuration (as in aryl-annulated phenazines), highlighting the structural precision enabled by the solution chemistry approach. Unsurprisingly XPS shows that the nitrogen dopants in the GQDs are "pyridinic", ${ }^{[33]}$ a form present in $\mathrm{N}$-doped more complex carbon materials with high
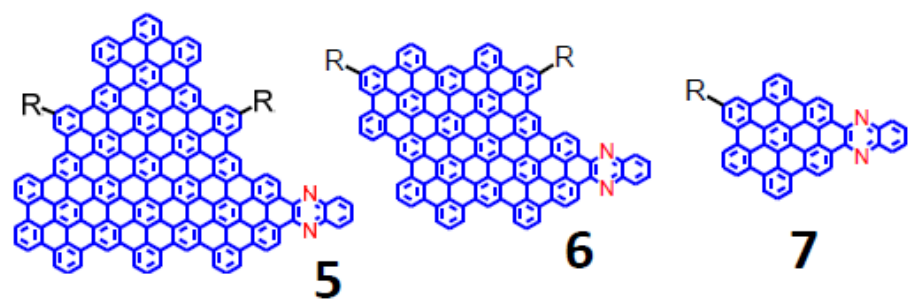

7

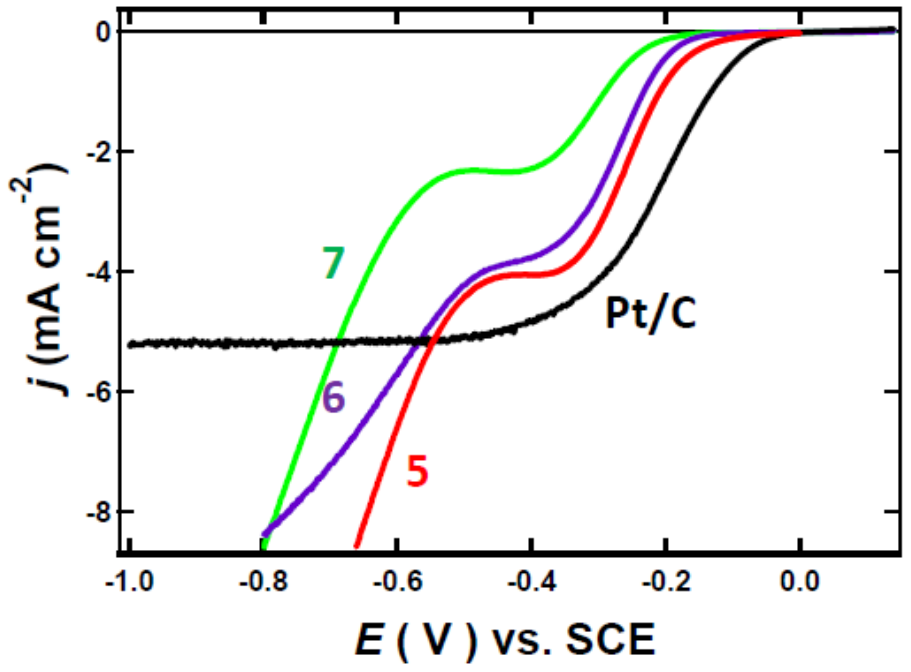

Figure 4. N-doped colloidal GQDs 5-7 and the LSV curves obtained for the GQDs deposited on a glassycarbon $\mathrm{RDE}$ in an $\mathrm{O}_{2}$-saturated $0.1 \mathrm{M} \mathrm{KOH}$ aqueous solution $(1600 \mathrm{rpm})$. (Adapted with permission from ref 32. Copyright 2012 American Chemical Society.)
ORR activity.

Electrochemical studies of the Ndoped GQDs not only showed electrocatalytic ORR activity but also revealed that the conjugation size in these materials was an important parameter determining the ORR activity. ${ }^{[32]}$ Figure 4 shows the linear sweep voltammetry (LSV) curves obtained for GQDs 5-7 and commercial Pt/C (20\% wt. Pt on Vulcan XC-72R) of the same mass deposited on a glassy-carbon rotating disk electrode (RDE) in an $\mathrm{O}_{2}$ saturated $\quad 0.1 \quad \mathrm{M} \quad \mathrm{KOH}$ aqueous solution (1600 rpm). GQDs 5-7 show ORR onset potentials at $-0.04,-0.10$, and $-0.14 \mathrm{~V}$, respectively, relative to that for the commercial Pt/C catalyst. In addition, the cathodic current density increases with size of the 
GQDs, indicating improved catalytic activity of larger GQDs. Remarkably our results contradict the common belief in the carbon catalysis community that higher nitrogen content leads to higher catalytic activity. Rather, the largest N-doped GQD 1 studied here, with the lowest doping level, shows the highest activity. This should make us reconsider experimental strategies to improve the activity of carbon materials. Traditional approaches that focus on increasing the content of nitrogen atoms with a specific bonding configuration alone may not be sufficient.

An important question not understood in the ORR community has been why $\mathrm{N}$-doped graphitic carbon materials can electrocatalytically activate the kinetically inert oxygen at ambient temperature. To help answer this question, we conducted combined electrochemical and computational studies on the N-doped GQDs. ${ }^{[33]}$ Shown in Figure 5a are cathodic polarization curves obtained with a solid film of 7 on a glassy-carbon electrode in, respectively, an $\mathrm{O}_{2-}$ saturated (solid curve, left axis) and an $\mathrm{O}_{2}$-free (argon-saturated, dotted curve, right axis) $0.1 \mathrm{M}$ $\mathrm{KOH}$ aqueous solution ( $\mathrm{pH} 13.0$ ). In the solid curve the shoulder near $-0.4 \mathrm{~V}$ is attributed to the
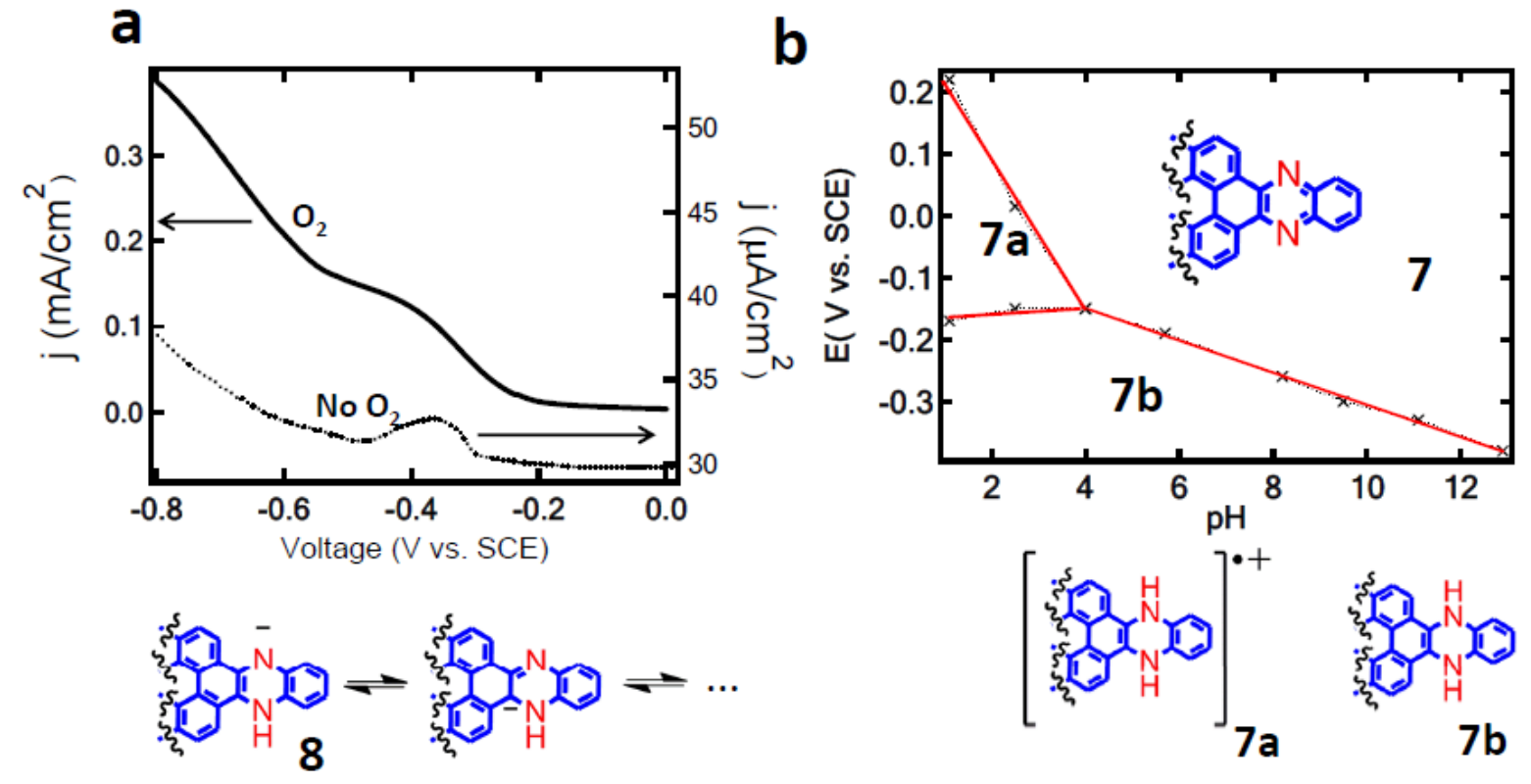

Figure 5. Electrochemical reduction of 7 and ORR activity. (a) Cathodic polarization curves obtained with a solid film of $\mathbf{7}$ on a glassy-carbon electrode in, respectively, an $\mathrm{O}_{2}$-saturated (solid curve, left axis) and an $\mathrm{O}_{2}$-free (argon-saturated, dotted curve, right axis) $0.1 \mathrm{M} \mathrm{KOH}$ aqueous solution. (b) Pourbaix diagram of 7 at $25^{\circ} \mathrm{C}$. Also shown are the proposed structures for the most stable species in each region of the diagram. (Adapted with permission from ref 33. Copyright 2014 American Chemical Society.) 
electrocatalytic reduction of $\mathrm{O}_{2}$. Remarkably, the reduction of 7 (without $\mathrm{O}_{2}$ ) occurs at the same potential yet with a current that is two orders of magnitude lower, suggesting that the reduction product(s) of $\mathbf{7}$ are responsible for catalytically activating the oxygen and initiating the ORR.

To understand the nature of the electrochemical reduction of 7 and to identify the species responsible for oxygen activation, we measured its reduction potential at $25^{\circ} \mathrm{C}$ and various $\mathrm{pH}$ values and constructed a Pourbaix diagram. ${ }^{[33]}$ The Pourbaix diagram, shown in Figure 5b, delineates the potential and $\mathrm{pH}$ ranges in which either 7 or its reduction products are thermodynamically stable. It clearly shows that reduction of $\mathbf{7}$ is $\mathrm{pH}$-dependent, indicating the participation of protons in the process. ${ }^{[34]}$

From the slopes of the lines in the Pourbaix diagram we can identify reduction products of 7 in alkaline electrolytes where 7 was shown to electrocatalyze the ORR. ${ }^{[33]}$ Between the $\mathrm{pH}$ values from 4 to 13 , the reduction potential varies with $\mathrm{pH}$ with a slope of $-27 \mathrm{mV}$ per $\mathrm{pH}$ unit, consistent with a concerted one-proton, two-electron process. From stoichiometry we can determine that the product is an amide anion with structure $\mathbf{8}$ shown in Figure 5,

$$
\mathbf{7}+\mathrm{H}^{+}+2 e \rightarrow \mathbf{8} .
$$

Because of the delocalization of the negative charge over the conjugated system (some of the resonance structures of $\mathbf{8}$ are shown in Figure 5), $\mathbf{8}$ possesses the characters of both an amide anion and a carbanion. Due to its strong basicity, 8 in water will undergo subsequent protonation to yield the thermodynamically stable product $\mathbf{7 b}$, involving no electron transfer and hence is not registered electrochemically.

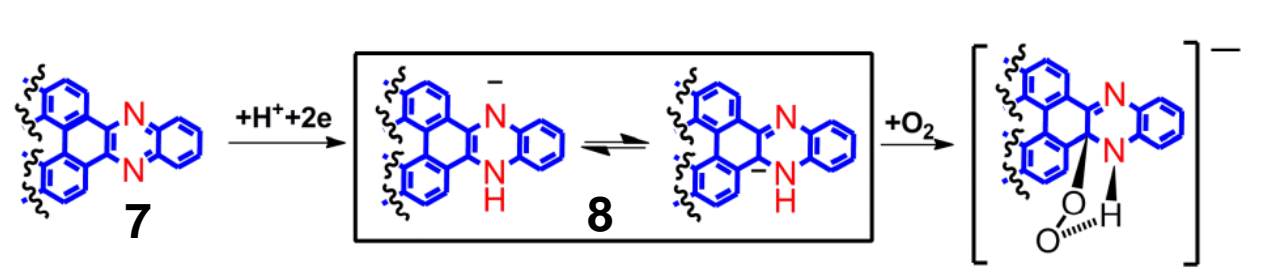

Figure 6. Proposed oxygen activation mechanism by $\mathbf{8}$. The oxygen activation occurs at a carbon atom next to the protonated nitrogen atoms. (Adapted with permission from ref 33. Copyright 2014 American Chemical Society.)
Our DFT calculations subsequently show that the anionic intermediate $\mathbf{8}$ is the intermediate that activates molecular oxygen. ${ }^{[33]}$ Figure 6

shows the key portion of the molecular structures in the process before and after the oxygen activation at a carbon atom next to the protonated nitrogen atoms (marked by the "-" sign in the second resonance structure of $\mathbf{8}$ in the rectangle). The product is essentially an organic peroxide 
with no net spin, more stable by $-0.09 \mathrm{eV}$ in vacuum and $-0.39 \mathrm{eV}$ in an aqueous environment relative to the reactants. The hydrogen atom attached to the nitrogen migrates to the terminal oxygen, resulting in an $\mathrm{O}-\mathrm{H} \times \mathrm{N}$ intramolecular hydrogen bond and the formation of a fivemembered ring. The $\mathrm{O}-\mathrm{O}$ bond length in the adduct is $1.43 \AA$, significantly longer than that of a triplet oxygen molecule $(1.20 \AA)$, suggesting that electrons are effectively transferred to the antibonding orbitals of the dioxygen. The oxygen adducts will undergo further reductions to yield water as the final product. In contrast, our calculations confirmed that the covalent binding between $\mathbf{7 b}$ and the triplet dioxygen is thermodynamically unfavorable by more than $0.5 \mathrm{eV}$. This is consistent with the observation that 7 has no ORR activity at $\mathrm{pH}$ lower than 11 , whereas 7b according to the Pourbaix diagram is stable at $\mathrm{pH}$ values as low as 4 .

Our studies for the first time provide a molecular-level explanation for why most metal-free, N-doped carbon materials can't electrocatalyze the ORR in acidic electrolytes. This according to the carbanion mechanism is because the intermediate $(\mathbf{8})$ responsible for activating oxygen is prone to protonation. The protonation would be fast enough to overtake the oxygen activation at $\mathrm{pH}$ lower than 11, thus poisoning the catalysts. ${ }^{[33]}$ Because an acidic electrolyte (e.g., $\mathrm{pH}=1$ ), due to its long-term tolerance to ambient $\mathrm{CO}_{2}$, is desired for current polymer exchange membrane fuel cell technology, ${ }^{[1,2]}$ this result implies that to make successful metal-free ORR catalysts for fuel cells we will need to make the active intermediate more resistant to protonation.

Thus our studies on the N-doped GQDs so far revealed that they could electrocatalyze the ORR because $\mathrm{N}$-doping enables two-electron reduction of the materials. The reduced carbon subsequently donates the two electron to oxygen, bypassing the otherwise thermodynamically unfavored superoxide formation. ${ }^{[34]}$ The overpotential of the catalyzed ORR is determined by the generation of the carbanion intermediates that activate oxygen. Interestingly the carbanion mechanism we proposed bears remarkable resemblance to the oxygen activation mechanism of flavin, a common cofactor for enzymes that catalyze one- and two-electron transfer reactions in aerobic metabolism. ${ }^{[35-38]}$ Consisting of a substituted quinoxaline fused with a uracil, the oxidized form of flavin can go through a two-electron reduction to yield a fully reduced form, which being an anion in biological conditions is known to activate oxygen by forming a hydroperoxylflavin intermediate. This naturally raises the question whether two-electron reduction is a general requirement for oxygen activation by carbon materials. Our research group 
is currently working to answer this question, as two-electron reduction is very common for $\mathrm{N}$ containing heterocyclic aromatic compounds. ${ }^{[39]}$

Studying the well-defined GQDs provides important mechanistic insights into carbon catalysis. Indeed, the multi-step synthesis currently required for making the GQDs suggests that the GQDs are too costly to be directly applied for any applications. Unless more efficient and simplified synthetic methods can be developed, currently we believe the merits of these studies lie in the guidance they can offer for improving activity of complex carbon materials. It was recently demonstrated that making aryl-annulated phenazine structures similar to those in GQDs 5-7 on a pre-treated glassy-carbon electrode could enable excellent ORR activity. ${ }^{[40]}$

\section{Study Chemical Reactions of Carbon Materials}

The readily availability of graphitic carbon materials makes them excellent precursors for new materials with novel properties. For example, converting $\mathrm{sp}^{2}$-hybridized carbon atoms to $\mathrm{sp}^{3}$-carbon leads to significantly different chemical and physical properties, of which early examples include graphitic oxide (also known as "graphene oxide") ${ }^{[41,42]}$ and graphite fluoride. ${ }^{[43,44]}$ Recent emergence of graphene containing one or few atomic layers has stimulated further interest in this approach as a means either to mass produce graphene or to control their properties for various potential applications. ${ }^{[45-47]}$ However, there are significant challenges in understanding the reaction mechanisms of the $\mathrm{sp}^{2}$-to-sp $\mathrm{sp}^{3}$ conversion within these large conjugated systems. Whereas knowledge and understanding of small aromatic compounds often provides important insights, the large conjugation size in graphitic carbon materials has been known to enable novel reactivity. For example, it has been demonstrated that, due to its disappearing HOMO-LUMO gap, bulk graphene can participate in the Diels-Alder reaction at moderate temperatures either as a diene or as a dienophile to result in cycloaddition in the basal plane. ${ }^{[48,49]}$ To more efficiently explore novel chemistry and better control the properties of graphitic carbon materials, it is imperative to conduct mechanistic studies on reactions involving these large conjugated systems.

We recently used well-defined GQDs as a model system to study the mechanism of graphene fluorination by $\mathrm{XeF}_{2}{ }^{[50]}$ This reaction is very attractive because it can introduce a finite bandgap to the zero-bandgap graphene under mild reaction conditions by producing $\mathrm{sp}^{3}$-carbon in the basal plane. ${ }^{[51,52]}$ In addition, the fluorination product can be defluorinated to recover graphene, which, when combined with lithographic techniques, can lead to graphene nanostructures with 
controlled patterns and dimensions. ${ }^{[53,54]}$ However, challenges in understanding the fluorination mechanisms within such large conjugated systems have prevented control of the graphene nanostructures with atomic precision. This reaction is generally assumed to involve atomic fluorine (i.e., free radical) that forms due to $\mathrm{XeF}_{2}$ decomposition. ${ }^{[51]}$ In contrast, small aromatic hydrocarbons such as benzene, naphthalene, pyrene, etc., have been shown to react with $\mathrm{XeF}_{2}$, a strong oxidant, through a radical cation mechanism, resulting in fluorination though only on the edge and mostly maintaining the $\mathrm{sp}^{2}$-hybridization of the carbon atoms. ${ }^{[55]}$ Thus by studying well-defined GQDs we

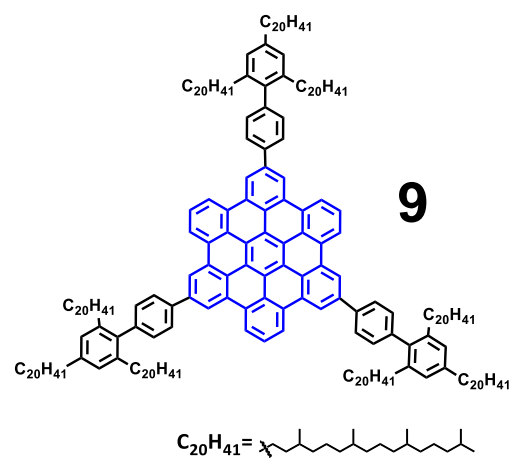
aimed to understand the origin of this apparent size-related difference in reactivity. A mechanistic understanding of this reaction meanwhile may lead to novel methods to control the functionalization of graphene for various applications.

For the fluorination studies 9 was synthesized with solution chemistry, and electron paramagnetic resonance spectroscopy (EPR) revealed at least three kinds of radical cations in a reaction solution containing 9 and $\mathrm{XeF}_{2}$ (Figure 7). ${ }^{[50]}$ Most notably a double-doublet hyperfine splitting pattern is consistent with radical cations with two fluorine atoms added. And numerical analysis of the EPR spectra over time revealed a series of oxidation and fluorination reactions,

$$
\begin{aligned}
& \mathbf{9}-1 e \rightarrow \mathbf{9}^{+}, \\
& \mathbf{9} \cdot^{+}+F^{-} \rightarrow[\mathbf{9}-F] \cdot, \\
& {[\mathbf{9}-F] \cdot-1 e \rightarrow[\mathbf{9}-F]^{+},} \\
& {[\mathbf{9}-F]^{+}+F^{-} \rightarrow[\mathbf{9}-2 F],} \\
& {[\mathbf{9}-2 F]-1 e \rightarrow[\mathbf{9}-2 F]{ }^{+}, \ldots .}
\end{aligned}
$$



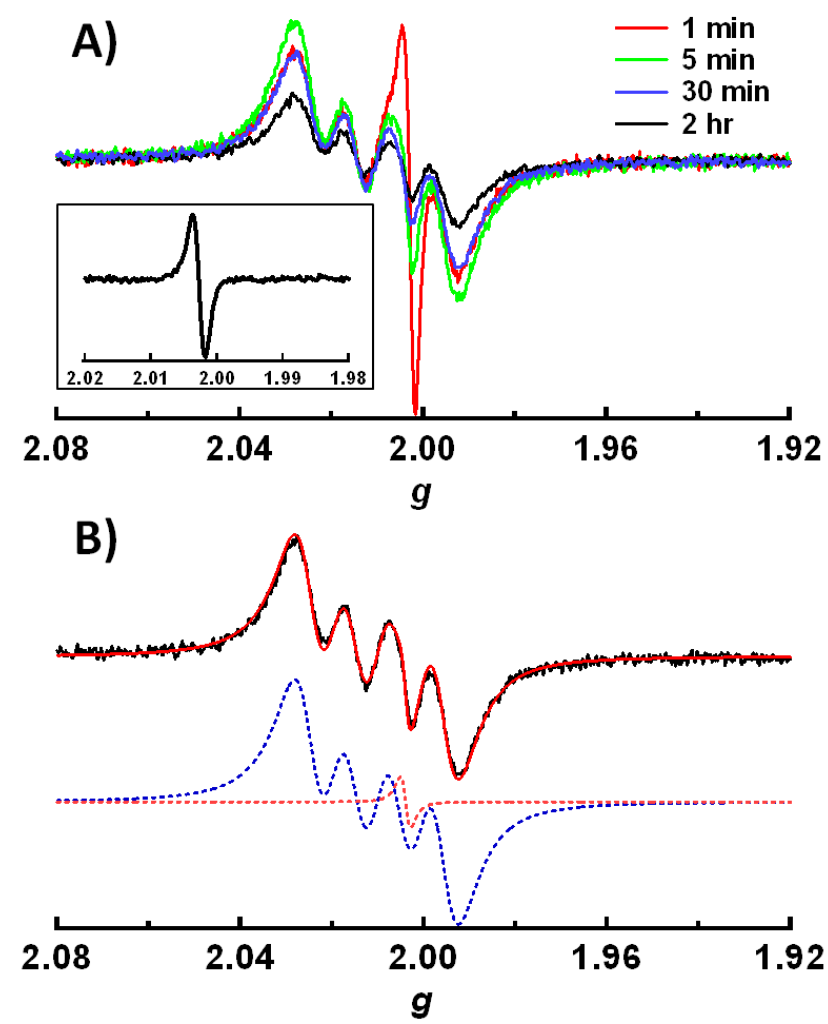

Figure 7. A) Room temperature EPR spectra of a reaction mixture at different reaction times $\left(\mathrm{XeF}_{2} / \mathbf{9}\right.$ molar ratio: 210:1). A sharp singlet can be readily seen in the 1-minute spectrum. In the later spectra a different singlet can be discerned at a slightly higher g-value with much lower amplitude. Inset: EPR spectrum in the early stage of the reaction measured at $250 \mathrm{~K}$, showing a singlet at $\mathrm{g}=2.0029$ and width of 5.0 Gauss. B) The 30-minute spectrum shown in Fig. 2A (black curve) and the simulated one (red solid curve) containing a set of double-doublets (blue dotted curve) and a singlet (red dotted curve). The singlet appears at $\mathrm{g}=2.0041$. (Adapted with permission from ref 50. Copyright 2015 American Chemical Society.)

Because a large number of reactions may occur in the reaction mixture, DFT calculations were carried out to help assign the observed hyperfine splitting patterns. ${ }^{[50]}$ We theoretically screened fluorine hyperfine splitting constants in neutral or cationic radical species of various possible fluorinated products of $\mathbf{9}$, and identified the structures consistent with the observed double-doublets in EPR (Figure 8a). They include difluoro addition (with two $\mathrm{sp}^{3} \mathrm{C}-\mathrm{F}$ bonds) products depicted in Figure $8 \mathrm{a}$ and further fluorine substitution (with $\mathrm{sp}^{2} \mathrm{C}-\mathrm{F}$ bonds) products. The difluoro addition products contain a fluorine atom in the central phenyl ring, at a site marked by a solid circle (red or green, Figure 8a) or its equivalent by symmetry, and the other by an open circle of the same color. The calculated splitting constants, labeled in Figure 8a, are insensitive to trans- or cis-arrangement of the two fluorine atoms. All these combinations are in reasonable agreement with the experimental double-doublets. According to our calculations, further fluorine substitution of the difluoro addition products barely modifies the splitting constants of the two fluorine atoms and leads to an extra splitting smaller than 4 Gauss, contributing only to the broadening of the double-doublets. 
The observed preferential fluorine addition at the sites revealed by the EPR measurements is consistent with frontier molecular orbital (FMO) theory. ${ }^{[56,57]}$ Shown in Figure $8 b$ is the wave function of the $\beta$ LUMO of $9^{\bullet+}$ (we assume the unpaired electron has spin $\alpha$ ), the FMO in the nucleophilic addition reaction with $\mathrm{F}^{-}$to produce $[\mathbf{9}-F] \bullet$, the mono fluoro addition products, as we as the calculated electrostatic potential of $\mathbf{9}^{\bullet+}$ (Figure 8c). Clearly the central phenyl ring has the highest electron density and the most positive electrostatic potential, and thus should be the preferred site for the first fluorine addition. To understand the site preference in the second fluorine addition, in Figure $8 \mathrm{~d}$ we show the wave function of the LUMO of $[\mathbf{9}-F]^{+}$with a fluorine atom (marked by a red dot) in the central ring (red circle), the FMO in the fluoride addition reaction to produce $[9-2 F]$. The electron density evidently indicates the sites at which the second fluorine addition would most likely occur. The six sites along the green circle are consistent with those depicted in Figure 8a that give rise to the observed double-doublets in EPR (Figure 7).

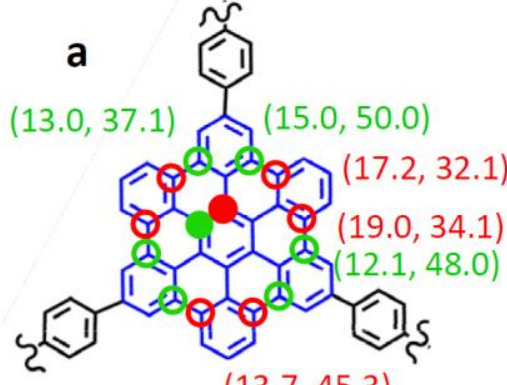

$(13.7,45.3)$

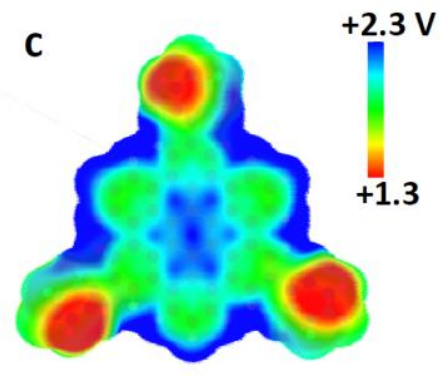

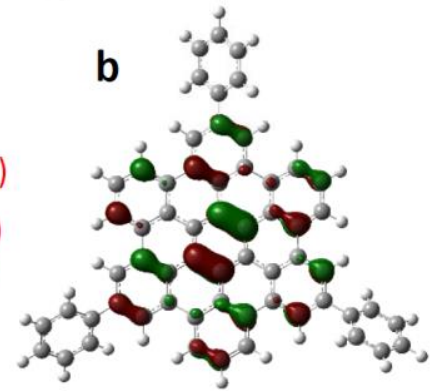

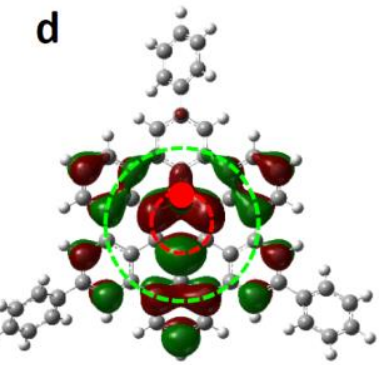

Figure 8. a) Combination of difluoro addition sites that according to our DFT calculations can yield the observed double-doublets in Fig. 7. One fluorine atom is located in the central phenyl ring, at a site marked by a solid circle (red or green) or its equivalent by symmetry, and the other by an open circle of the same color. The calculated hyperfine splitting constants of these pairs are also shown. b) $\beta$ LUMO of $9^{\circ+}$ as the FMO in the nucleophilic addition of $\mathrm{F}^{-}$to produce

$[9-F] \bullet$. c) Calculated electrostatic potential of $\mathbf{9}^{\bullet+}$. c) LUMO of $[\mathbf{9}-F]^{+}$as the FMO in the fluoride addition reaction to produce $[\mathbf{9}-2 F]$. (Adapted with permission from ref 50. Copyright 2015 American Chemical Society.)

Our study with combined spectroscopy techniques and theoretical calculated showed that graphene fluorination with $\mathrm{XeF}_{2}$ occurs via a radical cation mechanism leading to fluorination in the basal plane. With this mechanism and the FMO theory we can also understand the apparent size-related reactivity that no basal plane fluorination was previously observed with small aromatic hydrocarbons. 
Calculated FMO orbitals show that this is because the radical cations of previously studied small polycyclic aromatic hydrocarbon have low electron density in the basal plane. ${ }^{[6,57]}$

\section{Conclusions and Outlook}

Here we have presented a few examples where we used well-defined colloidal GQDs as model systems to understand fundamental processes in graphitic carbon materials. Such studies, though in the early stage, have provided useful insights regarding the functions of carbon in catalysis and mechanisms of chemical reactions in carbon materials. As shown above, essential to the studies is the synergy between experimental and computational investigations, which not only helps with the interpretation of experimental results but also reveals reactive intermediates and suggests possible reaction pathways.

We anticipate further work exploiting the synergy between experimental and computational investigations will lead to exciting opportunities in understanding and designing of carbon materials. In particular, an understanding of "structure sensitivity" ${ }^{[58,59]}$ in carbon catalysis is urgently needed. As has been demonstrated with well-defined metal and metal oxide single crystal surfaces ${ }^{[60-64]}$ a molecular level understanding of catalysis makes it possible to rationally design catalysts with improved reactivity and selectivity.

Another area where the GQDs can make significant contribution is biomedicine. Carbon materials such as nanotubes and graphene have been intensively studied for both in vitro and in vivo imaging, drug delivery, and cancer therapy. ${ }^{[65-67]}$ Because of their large absorption for nearIR radiation that can penetrate tissues, the carbon materials are especially attractive as potential photothermal or photodynamic agents in which the near-IR radiation triggers targeted cell destruction. Challenges persist, however, again because of poorly controlled structures of the carbon materials. It has been noted that the size, shape, as well as surface chemistry of the nanotubes or graphene affect the outcome of studies, leading to conflicting observations. ${ }^{[68]}$ The well-defined colloidal GQDs, if made water-soluble, could overcome these difficulties. GQDs with size greater than $2.5 \mathrm{~nm}$ (such as $\mathbf{1}$ and $\mathbf{3}$ shown in Figure 1) have significant near-IR absorption cross-section. ${ }^{[12]}$

Lastly, it is important to realize that there are limitations to using the well-defined GQDs as model systems. For example, processes such as photoluminescence, electron transfer, and carrier cooling that involve graphitic carbon materials in their excited states have recently been studied 
for light harvesting, photocatalysis, bioimaging, and sensing purposes. ${ }^{[10,65,69-74]}$ Because energy relaxation processes in conjugated systems can be significantly altered by environment, caution should be exercised to translate those observed in the GQDs to more complex systems. ${ }^{[75]}$ In addition, curvature and defects widely present in carbon materials cannot be modeled with the nearly planar GQDs. Thus the GQDs are only the "zeroth-order approximation" focusing on the conjugated framework and dopants. Nevertheless we hope this will stimulate further efforts toward unraveling important processes in carbon materials for sustainable technologies.

\section{Acknowledgement}

We thank Professors Krishnan Raghavachari and Allen Siedle at Indiana University and Professor Liming Dai at Case Western Reserve University for collaborations and helpful discussions on work discussed herein. This work was supported by the National Science Foundation . 


\section{References}

[1] Wu G, Zelenay P. Nanostructured nonprecious metal catalysts for oxygen reduction reaction. Acct Chem Res 2013:46;1878-89.

[2] Yu D, Nagelli E, Du F, Dai L. Metal-free carbon nanomaterials become more active than metal catalysts and last longer. J Phys Chem Lett 2012:1;2165-73.

[3] Yang, Z., Ren J, Zhang Z, Chen X, Guan G, Qiu L, Zhang Y, Peng H. Recent advancement of nanostructured carbon for energy applications. Chem Rev 2015:115;5159-223.

[4] Wang QH, Jin Z, Kim KK, Hilmer AJ, Paulus GLC, Shih C-J, Ham M-H, Sanchez-Yamagishi JD, Watanabe K, Taniguchi T, Kong J, Jarillo-Herrero P, Strano S. Understanding and controlling the substrate effect on graphene electron-transfer chemistry via reactivity imprint lithography. Nature Chem 2012:4:724-32.

[5] Levy N, Burke SA, Meaker KL, Panlasigui M, Zettl A, Guinea F, Castro Neto AH, Crommie MF. Strain-induced pseudo-Magnetic fields greater than 300 Tesla in graphene nanobubbles. Science 2010:329;544-7.

[6] Klimov NN, Jung S, Zhu S, Li T, Wright CA, Solares SD, Newell DB, Zhitenev NB, Stroscio JA. Electromechanical properties of graphene drumheads. Science 2012:336;1557-61.

[7] Bandosz TJ. Surface Chemistry of Carbon Materials. In Carbon Materials for Catalysis. Serp P, Figueiredo JL. Eds. John Wiley \& Sons. 2009:;45-91.

[8] Liu H, Liu Y, Zhu D. Chemical doping of graphene. J Mat Chem 2011:21;3335-45.

[9] Wang H, Maiyalagan T, Wang X. Review on recent progress in nitrogen-doped graphene: Synthesis, characterization, and its potential applications. ACS Catalysis 2012:2;781-95.

[10] Yan X, Li B, Li L-S. Colloidal graphene quantum dots with well-defined structures. Acct Chem Res 2013:46;2254-62.

[11] Li L-S, Yan X. Colloidal graphene quantum dots. J Phys Chem Lett 2010:1;2572-6.

[12] Yan X, Cui X, Li L-S. Synthesis of large, stable graphene quantum dots with tunable size. J Am Chem Soc 2010:132;5944-5.

[13] Yan X, Li L-S. Solution-chemistry approach to graphene nanostructures. J Mat Chem 2011:21;3295-300.

[14] Yan X, Cui X, Li B, Li L-S. Large, solution-processable graphene quantum dots as light absorbers for photovoltaics. Nano Lett 2010:10;1869-73.

[15] Bitter JH, De Jong KP. Preparation of carbon-supported metal catalysts. In Carbon Materials for Catalysis. Serp P, Figueiredo JL. Eds. John Wiley \& Sons, 2009:;157-76.

[16] Rodriguez-Reinoso F, Sepulveda-Escribano A. Carbon as catalyst support. In Carbon Materials for Catalysis. Serp P, Figueiredo JL, Eds. John Wiley \& Sons. 2009:;131-55. 
[17] Kauffman DR, Sorescu DC, Schofield DP, Allen BL, Jordan KD, Star A. Understadning the sensor response of metal-decorated carbon nanotubes. Nano Lett 2010:10;958-63.

[18] Zan R, Bangert U, Ramasse Q, Novoselov KS. Metal-graphene interaction studies via atomic resolution scanning transmission electron microscopy. Nano Lett 2011:11;1087-92.

[19] Toebes ML, van Dillen JA, de Jong KP. Synthesis of supported palladium catalysts. J Mol Cata A 2001:17;375-98.

[20] Kamat PV. Graphene-based nanoarchitectures. Anchoring semiconductor and metal nanoparticles on a two-dimensional carbon support. J Phys Chem Lett 2010:1;520-7.

[21] Wildgoose GG, Banks CE, Compton RG. Metal nanoparticles and related materials supported on carbon nanotubes: Methods and applications. Small 2006:2;182-93.

[22] Chan KT, Neaton JB, Cohen ML. First-principles study of metal adatom adsorption on graphene. Phys Rev B 2008:77;235430.

[23] Wang QJ, Che JG. Origins of distinctly different behaviors of Pd and Pt contacts on graphene. Phys Rev Lett 2009:103;066802.

[24] Yan X, Li Q, Li L-S. Formation and stabilization of palladium nanoparticles on colloidal graphene quantum dots. J Am Chem Soc 2012:134;16096-8.

[25] Chen Z, Appenzeller J, Knoch J, Lin Y, Avouris P. The role of metal-nanotube contact in the performance of carbon nanotube field-effect transistors. Nano Lett 2007:5;1497-502.

[26] Mann D, Javey A, Kong J, Wang Q, Dai H. Ballistic transport in metallic nanotubes with reliable Pd ohmic contacts. Nano Lett 2003:3;1541-4.

[27] Figueiredo JL, Pereira MFR. Carbon as catalyst. In Carbon Materials for Catalysis. Serp P, Figueiredo JL. Eds. John Wiley \& Sons. 2009:;177-217

[28] Knoblauch K, Richter E, Jüntgen H. Application of active coke in processes of SO2- and NOxremoval from flue gases. Fuel 1981:60;832-8.

[29] Zhang J, Liu X, Blume R, Zhang A, Schlögl R, Su DS. Surface-modified carbon nanotubes catalyze oxidative dehydrogenation of n-butane. Science 2008:322;3-7.

[30] Zhang J, Wang X, Su Q, Zhi L, Thomas A, Feng X, Su DS, Schlögl R, Mullen K. Metal-free phenanthrenequinone cyclotrimer as an effective heterogeneous catalyst. J Am Chem Soc 2009:131;11296-7.

[31] Gammon WJ, Kraft O, Reilly AC, Holloway BC. Experimental comparison of N(1s) x-ray photoelectron spectroscopy binding energies of hard and elastic amorphous carbon nitride films with reference organic compounds. Carbon 2003:41;1917-23. 
[32] Li Q, Zhang S, Dai L, Li L-S. Nitrogen-doped colloidal graphene quantum dots and their sizedependent electrocatalytic activity for the oxygen reduction reaction. J Am Chem Soc 2012:134;18932-5.

[33] Li Q, Noffke B, Wang Y, Menezes B, Peters DG, Raghavachari K, Li L-S. Electrocatalytic oxygen activation by carbanion intermediates of nitrogen-doped graphitic carbon. J Am Chem Soc 2014:136;3358-61.

[34] Ho RYN, Liebman JF, Valentine JS. Overview of the Energetics and Reactivity of Oxygen. In Active Oxygen in Chemistry. 1st ed. Foote CS, Valentine JS, Greenberg A, Liebman JF. Eds. Chapman \& Hall: London. 1996:2;1-23.

[35] Silverman RB. The Organic Chemistry of Enzyme-Catalyzed Reactions. Academic Press: London. 2002.

[36] Bruice TC. Mechanisms of flavin catalysis. Acct Chem Res 1980:13;256-62.

[37] Ghisla S, Massey V. Mechanisms of flavoprotein-catalyzed reactions. Eur J Biochem 1989:181;117.

[38] Palfey BA, Ballou DP, Massey V. Oxygen activation by flavins and pterins. In Active Oxygen in Biochemistry. Valentine JS, Foote CS, Greenberg A, Liebman JF. Eds. Chapman \& Hall: London. 1995:;37-83.

[39] Grimshaw J. Electrochemical Reactions and Mechanisms in Organic Chemistry. Elsevier: Amsterdam. 2000.

[40] Fukushima T, Drisdell W, Yano J, Surendranath Y. Graphite-conjugated pyrazines as molecularly tunable heterogeneous electrocatalysts J Am Chem Soc 2015:137;10926-9.

[41] Brodie BC. On the atomic weight of graphite. Phil Trans R Soc Lond 1859:149,249-59.

[42] Hummers WS, Offeman RE. Preparation of graphitic oxide. J Am Chem Soc 1958:80;1339-9.

[43] Palin DE, Wadsworth KD. Structure of carbon monofluoride Nature 1948:162;925-6.

[44] Ruff O, Bretschneider O. Die Reaktionsprodukte der verschiedenen Kohlenstoffformen mit Fluor II (Kohlenstoff'monofluorid). Z Anorg Allg Chem 1934:217;1-18.

[45] Dreyer DR, Park S, Bielawski CW, Ruoff RS. The chemistry of graphene oxide. Chem Soc Rev 2010:39;228-40.

[46] Rodriguez-Perez L; Herranza MA, Martin N. The chemistry of pristine graphene. Chem Comm 2013:49;3721-35.

[47] Sarkar S, Bekyarova E, Haddon RC. Covalent chemistry in graphene electronics. Materialstoday 2012:15;276-85.

[48] Sarkar S, Bekyarova E, Haddon RC. Chemistry at the Dirac point: Diels-Alder reactivity of graphene. Acct Chem Res 2012:4;673-82. 
[49] Sarkar S, Bekyarova E, Niyogi S, Haddon RC. Diels-Alder chemistry of graphite and graphene: Graphene as diene and dienophile. J Am Chem Soc 2011:133;3324-7.

[50] Liu Y, Noffke BW, Qiao X, Li Q, Gao X, Raghavachari K, Li LS. Basal plane fluorination of graphene by $\mathrm{XeF}_{2}$ via a radical cation mechanism. J Phys Chem Lett 2015:6;3645-9.

[51] Nair RR, Ren W, Jalil R, Riaz I, Kravets VG, Britnell L, Blake P, Schedin F, Mayorov AS, Yuan S, Katsnelson MI, Cheng H-M, Strupinski W, Bulusheva LG, Okotrub AV, Grigorieva IV, Grigorenko AN, Novoselov KS, Geim A. Fluorographene: A two-dimensional counterpart of Teflon Small 2010:6;2877-84.

[52] Robinson JT, Burgess JS, Junkermeier CE, Badescu SC, Reinecke TL, Perkins FK, Zalalutdniov MK, Baldwin JW, Culbertson JC, Sheehan PE, Snow ES. Properties of fluorinated graphene films. Nano Lett 2010:10;3001-5.

[53] Lee W-K, Haydell M, Robinson JT, Laracuente AR, Cimpoiasu E, King WP, Sheehan PE. Nanoscale reduction of graphene fluoride via thermochemical nanolithography. ACS Nano 2013:7;6219-24.

[54] Lee W-K, Robinson JT, Gunlycke D, Stine RR, Tamanaha CR, King WP, Sheehan PE. Chemically isolated graphene nanoribbons reversibly formed in fluorographene using polymer nanowire masks. Nano Lett 2011:11;5461-4.

[55] Filler R. Reactions of organic compounds with xenon fluorides. Israel J Chem 1978:17;71-9.

[56] Fleming I. Molecular Orbitals and Organic Chemical Reactions: Reference Edition. Wiley \& Sons: Chichester. 2010.

[57] Fukui K. Theory of Orientation and Stereoselection. Springer: Berlin. 1975.

[58] Nørskov JK. Electronic factors in catalysis. Prog Surf Sci 1991:38;103-44.

[59] Nørskov JK, Bligaard T, Hvolbæk B, Abild-Pedersen F, Chorkendorffc I, Christensen CH. The nature of the active site in heterogeneous metal catalysis. Chem Soc Rev 2008:37;2163-71.

[60] Somorjai GA, Li Y. Introduction to Surface Chemistry and Catalysis; Wiley: Hoboken, NJ. 2010.

[61] Goodman DW. Model studies in catalysis using surface science probes. Chem Rev 1995:xx;52336.

[62] Goodman DW. Correlations between surface science models and "real-world" catalysts. J Phys Chem 1996:100;13090-102.

[63] Ertl G. Reactions at curfaces: From atoms to complexity. Angew Chem Intl Ed 2008:47;3524-35.

[64] Somorjai GA, Li Y. Major successes of theory-and-experiment-combined studies in surface chemistry and heterogeneous catalysis. Top Catal 2010:53;311-25.

[65] Luo PG, Sahu S, Yang S-T, Sonkar SK, Wang J, Wang H, LeCroy GE, Cao L, Sun YP. Carbon “quantum" dots for optical bioimaging. J Mat Chem B 2013:1;2116-27. 
[66] Liu Z, Robinson JT, Tabakman SM, Yang K, Dai H. Carbon materials for drug delivery and cancer therapy. Materialstoday 2011:14;316-23.

[67] Yang K, Feng L, Shi X, Liu Z. Nano-graphene in biomedicine: Theranostic applications. Chem Soc Rev 2013:42;530-47.

[68] Kostarelos K, Novoselov KS. Exploring the interface of graphene and biology. Science 2014:344;261-3.

[69] Fernando KAS, Sahu S, Liu Y, Lewis WK, Guliants EA, Jafariyan A, Wang P, Bunker CE, Sun Y-P. Carbon quantum dots and applications in photocatalytic energy conversion. ACS Appl Mater. Interfaces 2015:7;8363-76.

[70] Baker SN, Baker GA. Luminescent carbon nanodots: Emergent nanolights. Angew Chem Intl Ed 2010:49;6726-44.

[71] Mueller ML, Yan X, McGuire JA, Li L-S. Triplet states and Electronic relaxation in photoexcited graphene quantum dots. Nano Lett 2010:10;2679-82.

[72] Mueller ML, Yan X, Dragnea B, Li LS. Slow hot-carrier relaxation dynamics in colloidal graphene quantum dots. Nano Lett 2011:11;56-60.

[73] Sun C, Figge F, McGuire JA, Li Q, Li L-S. Biexciton Auger recombination in colloidal graphene quantum dots. Phys Rev Lett 2014:113;107401

[74] Sun C, Figge F, Ozfidan I, Korkusinski M, Yan X, Li L-S, Hawrylak P, McGuire JA. Biexciton binding of Dirac fermions confined in colloidal graphene quantum dots. Nano Lett 2015;15:5472-6.

[75] Dekaliuk MO, Viagin O, Malyukin YV, Demchenko AP. Fluorescent carbon nanomaterials: “quantum dots” or nanoclusters? Phys Chem Chem Phys 2014;16:16075-84. 